\title{
GMR
}

\section{Targeted gene therapy and in vivo bioluminescent imaging for monitoring postsurgical recurrence and metastasis in mouse models of liver cancer}

\author{
Q. He*, C.L. Yao*, L. Li, Z. Xin, Z.K. Jing and L.X. Li \\ Department of Hepatic, Biliary, Pancreatic, and Splenic Surgery, \\ Beijing Chaoyang Hospital of Capital Medical University, Beijing, China \\ *These authors contributed equally to this study. \\ Corresponding author: L.X. Li \\ E-mail: xqhdoc@163.com
}

Genet. Mol. Res. 15 (3): gmr.15037878

Received October 21, 2015

Accepted March 11, 2016

Published August 12, 2016

DOI http://dx.doi.org/10.4238/gmr.15037878

Copyright (C) 2016 The Authors. This is an open-access article distributed under the terms of the Creative Commons Attribution ShareAlike (CC BY-SA) 4.0 License.

\begin{abstract}
We investigated the effects of combined targeted gene therapy on recurrence and metastasis after liver cancer resection in nude mice. Twenty BALB/C mice were randomly divided into control and treatment groups with 10 mice in each group and a male/female ratio of 1:1. Luciferase gene-labeled human primary hepatic carcinoma cell line MHCC97-H was then used to prepare a carcinoma model. An optical in vivo imaging technique (OIIT) was used 10 days later to detect the distribution of tumor cells, followed by partial liver resection and gene therapy. In the treatment group, $100 \mu \mathrm{L}$ phosphate-buffered saline (PBS) containing $1 \times 10^{12} \mathrm{rAAV} / \mathrm{AFP} / \mathrm{IL}-24$ gene viral vectors was injected into liver sections and peritumoral posterior peritoneal tissues; in the control group, the same amount of PBS containing $1 \mathrm{x}$ $10^{12}$ empty viral vectors was injected at the same sites. OIIT was then used to detect the in vivo tumor metastasis 21 days later. Luciferase
\end{abstract}


gene-labeled human primary hepatic carcinoma cell line MHCC97-H successfully infected 20 nude mice, and OIIT showed that the two groups exhibited metastasis after local tumor resection, but there were more tumor cells in the control group $(\mathrm{P}<0.05)$. $\mathrm{rAAV} / \mathrm{AFP} / \mathrm{IL}-24$ gene therapy can inhibit recurrence after liver cancer resection.

Key words: Hepatocellular carcinoma; Recurrence and metastasis; Combined gene therapy

\section{INTRODUCTION}

Primary liver cancer is one of the world's most common malignant tumors. Besides conventional surgery, many techniques have been used to treat the disorder. These include chemotherapy in the 1980s, percutaneous absolute alcohol injection (Gervais and Arellano, 2011), the newly developed technique of hepatic arterial chemoembolization (Li et al., 2015a), radiofrequency ablation (Witczak-Malinowska et al., 2003), high-intensity focused ultrasound treatment (Zhang et al., 2011), cryotherapy (Yang et al., 2012), and laser therapy (Li et al., 2014a). Although these techniques have been used successfully in clinical applications, there is still a requirement for a specific treatment method for primary liver cancer, because the methods mentioned above are mostly palliative treatments, and the options for the systemic treatment of liver cancer remain limited (Li et al., 2014b). Furthermore, postoperative extrahepatic metastasis and the recurrence of remnant liver cancer further restrict clinical efficacy and result in a poor prognosis (Lyra-González et al., 2015). In addition to extrahepatic metastasis (Ruiz-Morales et al., 2014), intrahepatic metastasis may also occur in primary liver cancer, which significantly shortens the survival period of patients with the disease (Kim et al., 2015). Therefore, controlling metastasis after local liver cancer resection could be an effective means of improving the postoperative survival period (Xia et al., 2014). With the continuous development of molecular biology and an improved understanding of tumor pathogenesis, combined gene therapy for treating malignant tumors has become a major focus of interest (Sosman and Puzanov, 2009). Currently, surgical resection is still the most effective means of treating liver metastases; however, timely postoperative gene therapy is expected to become a novel method for controlling metastasis and recurrence in patients with extensive recurrence or in those who cannot tolerate resurgery (Shi and Huang, 2015).

The optical in vivo imaging technique (OIIT) is a recently developed molecular and genetic expression analysis and detection system that works as follows. In small mammals, reporter gene-luciferase gene expression produces a luciferase protein. This protein oxidizes the small molecule substrate luciferin in the presence of oxygen and magnesium ions, and some of the subsequent chemical energy is transformed into visible light and released. Sensitive equipment can quantitatively determine the number of emitted photons in vitro and convert them into images. By inserting the luciferase gene into the targeted gene promoter, this gene becomes the reporter gene, thereby enabling the in vivo monitoring of the targeted gene (Hoehn, 2015). The technique has considerable advantages over traditional detection methods (Elias et al., 2008).

Based on previous studies, in this study we applied OIIT combined with targeted gene therapy to monitor therapeutic effects during recurrence and metastasis following liver cancer resection in nude mice.

Genetics and Molecular Research 15 (3): gmr.15037878 


\section{MATERIAL AND METHODS}

\section{Animals and modeling}

We used $20 \mathrm{BALB} / \mathrm{C}$ mice (male/female ratio $=1: 1 ; 4-6$ weeks old; $18-20 \mathrm{~g}$ ). This study was carried out in strict accordance with the recommendations in the Guide for the Care and Use of Laboratory Animals of the National Institutes of Health. The animal use protocol was reviewed and approved by the Institutional Animal Care and Use Committee (IACUC) of Capital Medical University. The mice were purchased from the Animal Center of the Academy of Military Medical Sciences, and bred under specific-pathogen-free (SPF) conditions. A post-liver resection recurrence model has been established previously by our research group. After successfully preparing the model and in situ implantation of MHCC97-H cells for 10 days, the mice were subjected to OIIT. The mice were then randomly divided into a treatment group and a control group, with 10 mice in each group. In the treatment group, $\mathrm{rAAV} / \mathrm{AFP} / \mathrm{IL}-24$ gene viral vectors $(100 \mu \mathrm{L}$, containing $1 \times 10^{12}$ virus particles, kindly provided by Dr. Zhang Huixiang, New Zealand University, Australia) were injected into liver sections and peritumoral posterior peritoneal tissues. The control mice were injected with an identical volume of empty viral vectors at the same sites. Each group was subjected to OIIT to detect the recurrence and metastasis of liver tumors on the 10th day of model preparation and the 21st day of intraperitoneal injection of fluorescein.

\section{Hepatic cancer cell line and luciferase-labeled hepatic cancer cells}

An MHCC97-H hepatic carcinoma cell line that could be used for screening and identification, and could stably express luciferase, was established by Beyotime Biotechnology Co. Ltd. After amplification and purification of the luciferase gene-carrying plasmids, they were effectively transfected into the highly metastatic human liver cancer cell line MHCC97-H. The cell line was screened for high positive expression. When the MHCC97-H cells had been cultured to the logarithmic growth phase, we used trypsin for digestion, and resuspended them in a cell culture medium to prepare a single-cell suspension. We counted the cells to ensure the live cell ratio was greater than $98 \%$, recentrifuged the cell suspension $(1000 \mathrm{r} / \mathrm{min}$ for $3 \mathrm{~min})$, resuspended it in an appropriate amount of saline, and injected $5 \times 10^{6}$ cells $/ 0.2 \mathrm{~mL}$ into the liver of each nude mouse to prepare the models.

\section{Operating procedures}

Operations were performed using the OIIT (IVISTM50, purchased from Xenogen Co., USA) as follows: after intraperitoneally injecting fluorescein, the mice were anesthetized and placed on an obscure imaging platform. Maestro software was used to control the platform and obtain the appropriate orientation, and the lights were automatically turned on and off for background imaging of the light emitted by the mice in the absence of ambient light. This was followed by superimposition of the background image to show the location of in vivo light clearly, completing the imaging operation. Finally, Maestro software was used for imaging analysis.

\section{Statistical analysis}

All experimental data are reported as means \pm standard deviation, and we used the

Genetics and Molecular Research 15 (3): gmr.15037878 
Student $t$-test for analysis of significance.

\section{RESULTS}

\section{Screening and identification of MHCC97-H human hepatic cancer cells}

After amplification and purification of the luciferase gene-carrying plasmids, they were effectively transfected into the highly metastatic human liver cancer cell line MHCC97-H. The cell line was subjected to G418 drug screening for high positive expression. The detection of luciferase showed that the number of cells exhibited a significant linear correlation with the light-emission value $\left(r^{2}=0.9925\right.$, Figure 1$)$.

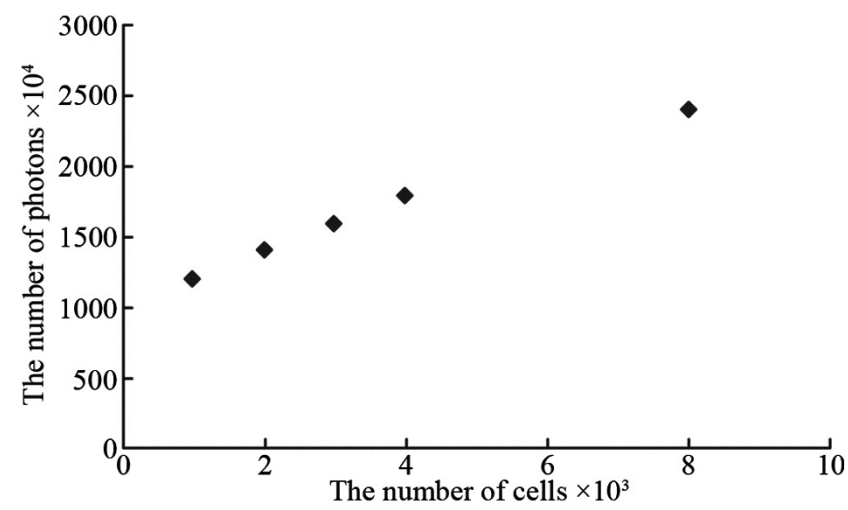

Figure 1. Near correlation between the strength of the emitted light and the number of labeled cells.

\section{OIIT of in situ transplantation model}

Ten days after in situ implantation of the MHCC97-H human hepatic cells, OIIT showed that the tumor cells had survived in the mice livers after surgery, and bio-luminescence signals were detected in $93 \%$ of the nude mice (Figure 2).

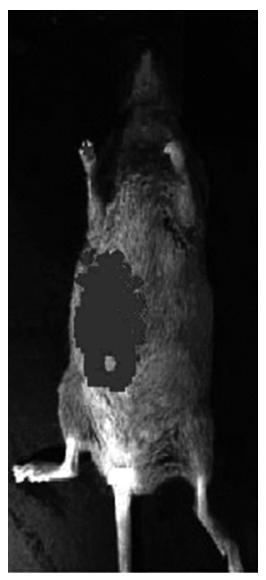

Figure 2. Image obtained using the optical in vivo imaging technique (OIIT) on the 10th day after surgery. 


\section{Detection of recurrence and metastasis}

The mice in each group were subjected to OIIT to detect recurrence and metastasis on the 21 st day after local liver cancer resection combined with the intraperitoneal injection of fluorescein. The results showed that on the 21 st day of gene therapy, the metastatic tumor cells in the liver and abdominal cavity clearly produced images (Figure 3A); more photons were detected in the control group, which indicated obvious tumor recurrence and metastasis, while the treatment group showed significantly fewer photons than the control group $(\mathrm{P}<0.05)$, indicating that when compared with the control group, the treatment effectively reduced the recurrence rate of liver cancer (Figure 3B).

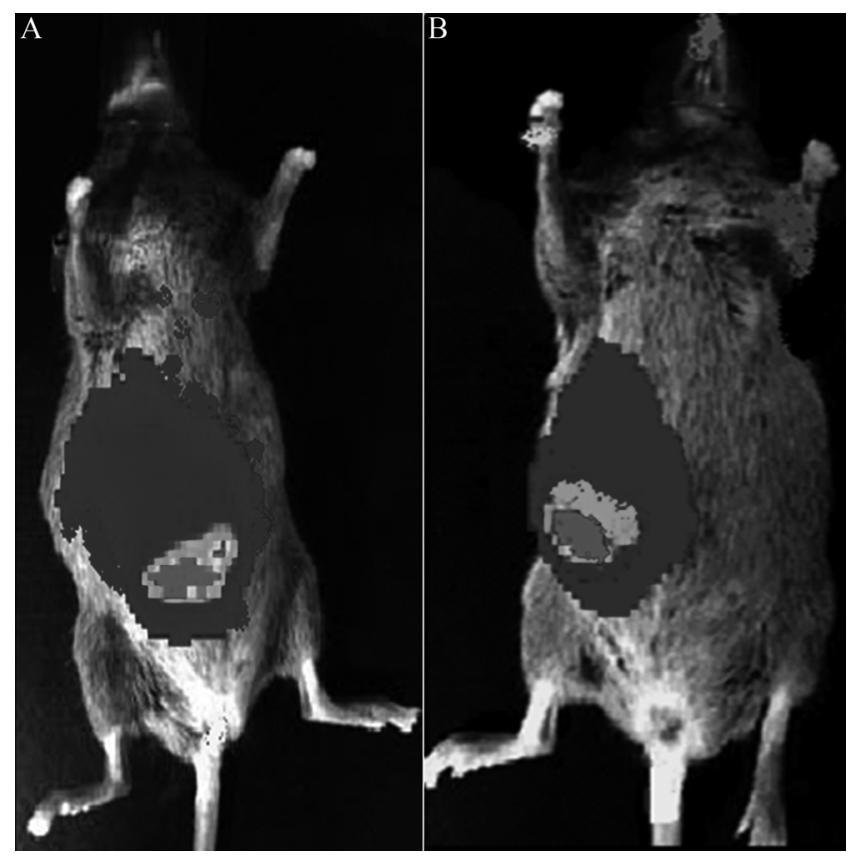

Figure 3. Comparison of the images obtained using the optical in vivo imaging technique (OIIT) on the 21 st day after liver cancer resection between the treatment group (A) and the control group (B).

\section{DISCUSSION}

Our research group has previously constructed an in situ transplantation model of liver cancer, and performed liver resection to explore the impact of gene therapy on recurrence and metastasis after liver cancer resection. We found that during construction of the liver cancer transplantation model, differences in the individual mice and the different sizes of the implanted tumors resulted in differences in model selection. Moreover, the treatment course could not be monitored in real time, and the effectiveness of the treatment could only be evaluated by killing the model animals. In the current study, OIIT was used to perform rapid, intuitive, and quantitative real-time monitoring, which avoided selection differences and allowed us to observe tumor growth at any time, so that scientific conclusions could be made on the treatment results (Kirschner et al., 2015). 
OIIT is a highly sensitive and intuitive imaging technology, and can directly monitor the activities of living cells using in vivo expression of a luciferase gene (Gervais and Arellano, 2011). It has the following advantages over conventional testing methods: 1) it is non-invasive; 2 ) it can be repeated at different time-points; 3 ) it can be used for fast scanning imaging (less than $5 \mathrm{~min}$ ); 4) it can generate the overall image of the experimental animal; and 5) it can perform real-time tracing of many important cells and molecules, especially tumor cells. OIIT can be used to observe growth, metastasis, and treatment activities inside live animal models, and represents a new approach to in vivo tumor research (Luker and Luker, 2008).

In this study, we transfected the luciferase gene into the highly metastatic human MHCC97-H hepatic cancer cell line, obtained the positive strains that exhibited high expression activities, and confirmed that the luciferase gene was stably expressed in the MHCC97-H cells. The luciferase-labeled cell lines were used to construct recurrence and metastasis nude mice models after liver cancer resection, and OIIT was used to observe the light-emitting signals from primary tumors and metastatic sites in real time. We found that the MHCC97-H cells still maintained high metastatic characteristics, several metastatic signals were found on the 10th day after in situ implantation, and the dynamic observation of signal changes indirectly reflected the growth of tumor cells in vivo. This study was much more accurate than previous studies that measured tumor volumes, and it reduced disturbances caused by peritumoral edema, as well as the necrosis of intratumoral cells and tissues. Therefore, it could be a good foundation for further experiments.

With the continuous development of molecular biology and an improved understanding of tumor pathogenesis, gene therapy has been used as a new strategy for the mainstream treatment of malignant tumors, and warrants further exploration. The premise of gene therapy in cancer should be to remove the primary lesions of a tumor maximally by using highly targeted gene therapy to kill residual micrometastases (Nicolaidou and Koufaris, 2015). Therefore, in this study we used nude mice for the resection of primary tumor lesions, and injected genes into local liver sections and the areas around the liver tumor. The aim was to use highly targeted gene therapy to kill the remaining micrometastases, as well as to block the recurrence and metastasis of tumors.

Studies have shown that angiostatin and IL-24 are closely related to the growth and metastasis of tumors, so they are currently being studied as targets for tumor suppressor genes (Kirstein et al., 2006; Kreis et al., 2008). In this study, we used recombinant adeno-associated virus-mediated targeting angiostatin and the IL-24 gene for combination therapy, and after establishing an in situ implantation nude mouse model with human hepatic cancer, liver cancer resection was performed to maximally simulate the primary tumor resection. Subsequently, combination gene therapy was carried out to observe the prevention and recurrence of tumor metastasis. Gene therapy comprises the transfer of one or more genes of interest, as well as their products, safely and effectively into target cells in vivo (Li et al., 2015b). In the present study, we applied OIIT, and the results showed that the luciferase gene could be inserted into DNA molecules to observe the results of gene therapy. The application of the luciferase gene as a reporter gene in this study proved that the combined gene could be continuously, efficiently, and tissue-specifically expressed inside experimental animals, and also exerted significant inhibitory effects on recurrence and metastasis following liver cancer resection.

Based on previous studies, this study preliminarily investigated the effects of OIIT combined with targeted gene therapy in preventing the recurrence and metastasis of liver cancer in nude mice after liver cancer resection. Our results indicate that compared with

Genetics and Molecular Research 15 (3): gmr.15037878 
conventional techniques, OIIT combined with gene therapy has certain advantages, and we anticipate its use in further studies.

\section{Conflicts of interest}

The authors declare no conflict of interest.

\section{ACKNOWLEDGMENTS}

Research supported by the Capital Medical Development Foundation (Project\#2005-3010).

\section{REFERENCES}

Elias DR, Thorek DL, Chen AK, Czupryna J, et al. (2008). In vivo imaging of cancer biomarkers using activatable molecular probes. Cancer Biomark. 4: 287-305.

Gervais DA and Arellano RS (2011). Percutaneous tumor ablation for hepatocellular carcinoma. AJR Am. J. Roentgenol. 197: 789-794. http://dx.doi.org/10.2214/AJR.11.7656

Hoehn M (2015). Imaging of neuro-inflammation: past, present and future. Georgian Med. News 243: 82-84.

Kim JM, Kwon CH, Joh JW, Park JB, et al. (2015). Intrahepatic metastasis is more risky than multiple occurrence in hepatocellular carcinoma patients after curative liver resection. Hepatogastroenterology 62: 399-404.

Kirschner S, Felix MC, Hartmann L, Bierbaum M, et al. (2015). In vivo micro-CT imaging of untreated and irradiated orthotopic glioblastoma xenografts in mice: capabilities, limitations and a comparison with bioluminescence imaging. J. Neurooncol. 122: 245-254. http://dx.doi.org/10.1007/s11060-014-1708-7

Kirstein MN, Moore MM and Dudek AZ (2006). Review of selected patents for cancer therapy targeting tumor angiogenesis. Recent Patents Anticancer. Drug Discov. 1: 153-161. http://dx.doi.org/10.2174/157489206777442269

Kreis S, Philippidou D, Margue C and Behrmann I (2008). IL-24: a classic cytokine and/or a potential cure for cancer? J. Cell. Mol. Med. 12 (6A): 2505-2510. http://dx.doi.org/10.1111/j.1582-4934.2008.00372.x

Li D, Kang J and Madoff DC (2014a). Locally ablative therapies for primary and metastatic liver cancer. Expert Rev. Anticancer Ther. 14: 931-945. http://dx.doi.org/10.1586/14737140.2014.911091

Li D, Kang J, Golas BJ, Yeung VW, et al. (2014b). Minimally invasive local therapies for liver cancer. Cancer Biol. Med. 11: $217-236$.

Li HL, Ji WB, Zhao R, Duan WD, et al. (2015a). Poor prognosis for hepatocellular carcinoma with transarterial chemoembolization pre-transplantation: retrospective analysis. World J. Gastroenterol. 21: 3599-3606. http://dx.doi. org/10.3748/wjg.v21.i12.3599

Li J, Yu Y, Wang J, Yan Z, et al. (2015b). Establishment of a novel system for the culture and expansion of hepatic stemlike cancer cells. Cancer Lett. 360: 177-186. http://dx.doi.org/10.1016/j.canlet.2015.02.006

Luker GD and Luker KE (2008). Optical imaging: current applications and future directions. J. Nucl. Med. 49: 1-4. http:// dx.doi.org/10.2967/jnumed.107.045799

Lyra-González I, Flores-Fong LE, González-García I, Medina-Preciado D, et al. (2015). MicroRNAs dysregulation in hepatocellular carcinoma: Insights in genomic medicine. World J. Hepatol. 7: 1530-1540. http://dx.doi.org/10.4254/ wjh.v7.i11.1530

Nicolaidou V and Koufaris C (2015). MicroRNA responses to environmental liver carcinogens: Biological and clinical significance. Clin. Chim. Acta 445: 25-33. http://dx.doi.org/10.1016/j.cca.2015.03.006

Ruiz-Morales JM, Dorantes-Heredia R, Chable-Montero F, Vazquez-Manjarrez S, et al. (2014). Bone metastases as the initial presentation of hepatocellular carcinoma. Two case reports and a literature review. Ann. Hepatol. 13: 838-842.

Shi Y and Huang A (2015). Effects of sorafenib on lung metastasis in rats with hepatocellular carcinoma: the role of microRNAs. Tumour Biol. 36: 8455-8463. http://dx.doi.org/10.1007/s13277-015-3565-1

Sosman J and Puzanov I (2009). Combination targeted therapy in advanced renal cell carcinoma. Cancer 115 (Suppl): 2368-2375. http://dx.doi.org/10.1002/cncr.24234

Witczak-Malinowska K, Zadrozny D, Studniarek M, Szurowska E, et al. (2003). Preliminary assessment of utility of radiofrequency ablation technique in treatment of primary hepatocellular carcinoma (HCC) in patients with hepatic cirrhosis. Med. Sci. Monit. 9 (Suppl 3): 68-72.

Xia F, Wu L, Lau WY, Li G, et al. (2014). Positive lymph node metastasis has a marked impact on the long-term survival of

Genetics and Molecular Research 15 (3): gmr.15037878 
patients with hepatocellular carcinoma with extrahepatic metastasis. PLoS One 9: e95889. http://dx.doi.org/10.1371/ journal.pone.0095889

Yang Y, Lu Y, Wang C, Bai W, et al. (2012). Cryotherapy is associated with improved clinical outcomes of sorafenib for the treatment of advanced hepatocellular carcinoma. Exp. Ther. Med. 3: 171-180.

Zhang Y, Zhao J, Guo D, Zhong W, et al. (2011). Evaluation of short-term response of high intensity focused ultrasound ablation for primary hepatic carcinoma: utility of contrast-enhanced MRI and diffusion-weighted imaging. Eur. $J$. Radiol. 79: 347-352. http://dx.doi.org/10.1016/j.ejrad.2010.06.039

Genetics and Molecular Research 15 (3): gmr.15037878 